\title{
Research on Ethernet Based on STM32F107 in Network Laser Marking
}

\author{
Hongjun $\mathrm{Li}^{1}$ \\ School of Mechanical Engineering and Automation \\ Wuhan Textile University \\ Wu Han,China \\ E-MAIL:lihongjun@foxmail.com \\ Jiujiang Yan $^{2}$ \\ School of Mechanical Engineering and Automation \\ Wuhan Textile University \\ Wu Han,China \\ E-MAIL:justneedyounow@163.com
}

\author{
Fujun $\mathrm{Shi}^{3}$ \\ School of Mechanical Engineering and Automation \\ Wuhan Textile University \\ Wu Han,China \\ E-MAIL:shifujun1989@163.com \\ Wei Chen ${ }^{4}$ \\ School of Mechanical Engineering and Automation \\ Wuhan Textile University \\ Wu Han,China \\ E-MAIL:wchen@wtu.edu.cn
}

\begin{abstract}
The transmission of marking data nowadays require a separate $\mathrm{PC}$ as a client at the receiving terminal in laser marking technology.This paper presents a new data receiving and state feedback solution. It is the industrial Ethernet that based on the composition of embedded network technology.The scheme integrates a network communication module which consists the MAC's STM32F107 micro controller and DM9161A PHY interface device.The module and laser control module and laser galvo scanner motion control module and other basic modules make up the laser marking unit of this system.Furthermore,the laser generator and galvo scanner is the executing agency that implements the networking of laser marking.The experiments show that the scheme can effectively solve the problem of data transmission in laser marking technology,and it can eventually finish the purpose of laser marking after data processing and conversion.The research results have a broad market prospect, and it has the great significance to the development of laser application fields.
\end{abstract}

Keywords-Laser marking;Embedded Ethernet;STM32F107; Galvo scanner;XY2-100 protocol

\section{INTRODUCTION}

Laser marking technology is one of important applications of laser technology in today's industrial production.With the constant enlargement of the application domain, its system stability,convenience and costs have been paid more and more attention.In today's industrial field,single marking equipment generally consists of PC machine,control board,control software,laser generator,vibration lenses and other components.This traditional combination machine has characteristics of high costs, low efficiency, poor convenience and can not adapt to the actual mass production.In recent years, the embedded technology that is based on STM32F107 has made the considerable development in the field of industrial control with characteristics like professional, streamlined system and so on.The processor is interconnect type, using the ARM7 architecture of the Cortex-M3 core.The Ethernet interface which is based on high-speed Ethernet PHY chip DM9161A is also widely used in embedded systems to realize the functions of remote communication,monitoring and data processing of the systems. This research adopts the micro-controller STM32F107 to realize the control function of single station marking machine, and combined with the Ethernet module to solve the problem of the communication between single-station machine laser marking machine and its host. The research can realize the marking machine's high stability and low cost composition and finally it can satisfy the demand of high efficiency production of complex industrial environment.

\section{THE OVERALL DESIGN OF SYSTEM AND WORKING} PRINCIPLE

\section{A. Overall design of system}

Galvo scanning laser marking system is generally composed of a laser generator,the control board,and the galvo scanner lens that integrate $\mathrm{X} / \mathrm{Y}$ galvo scanner motor,focusing lens,laser galvanometer driven electric circuit.The core of laser marking system is its control system.In the design,the control system is realized by marking control unit which mainly composed of a laser control module,motion control module,the laser vibrating mirror network communication module and alarm module.System principle block diagram is shown in Fig .1.

\section{B. Analysis of the working principle}

The STM32F107 micro controller in the system integrates MAC(Media Access Control acronym,that MAC sublayer protocol), and it connects the external PHY chip and RJ-45 seat through the RMII that is defined by IEEE 802.3 protocol.The connection can build a Ethernet network intercommunication platform for all kinds of applications.As a result, the host computer and embedded client will form a kind of master-slave network connection, and the marking control unit can transmit data to host via the network communication module. 
When the system works, the host computer will transmit the character data to the guest machine which has an unique MAC address. The guest machine receives the character data through its network module of marking control unit.The data will be digitized for 16 bits position control signals that XY2-100 protocol requires in its texts.And then the signals are transmitted to the laser motion control module. The module is used for

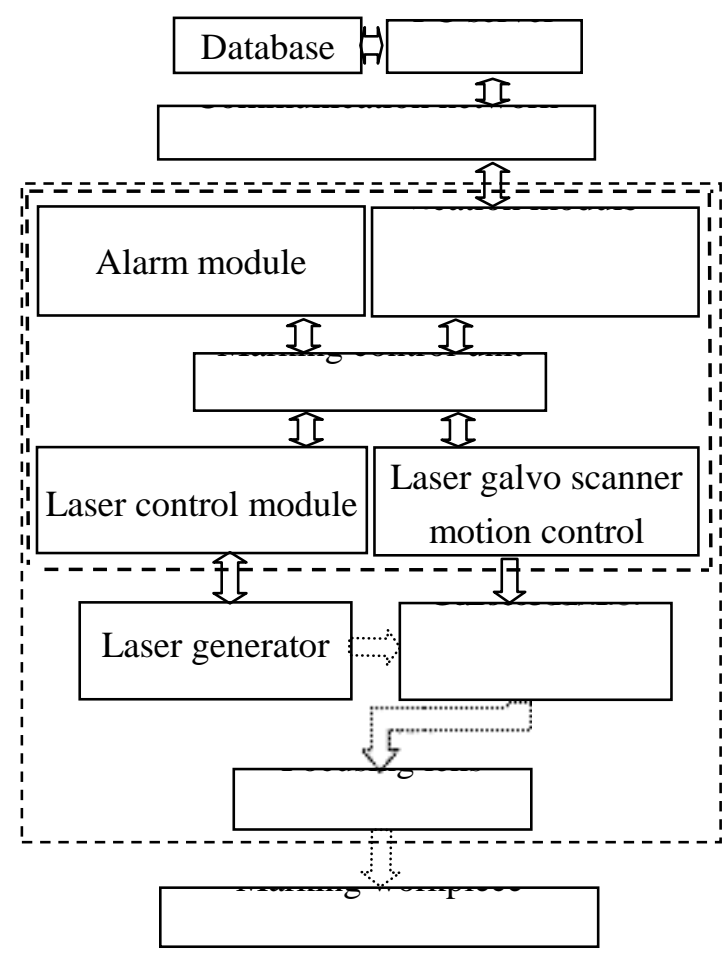

Figure 1. Network diagram of the principle of laser marking control system

differentiating, amplifying, comparing, closed-loop correcting and other processes to digital position control signals. Finally,it will be sent to the $\mathrm{X} / \mathrm{Y}$ galvo scanner motor and the motor rotates galvo scanner,then the galvo scanning laser engraving is realized.

\section{SYSTEM HARDWARE DESIGN}

\section{A. Hardware design of network communication module}

In the design, we choose STM32F107 chip as CPU.For one hand,it has rich hardware resources.Furthermore, it integrates Ethernet MAC and other important modules.Finally, speaking from the software,the micro-controller has sound standard library functions to facilitate the development of the system software.In addition,STM32F107 does not attaches to a physical layer device,so we choose DM9161A as the external PHY Ethernet network interface chip.The Ethernet data link layer MAC controller that STM32F107 provides in its hardware resource and the external physical layer interface chip and external auxiliary circuits composes of the high-speed Ethernet interface of the system.The system communicate with the host machine through the interface,and it can realizes the real-time receiving and sending of marking data and control signals though the receiving/transmitting register and $2 \mathrm{~K}$ FIFO cache(First In First Out) that are provided by CPU.The block diagram of the module is shown in Fig .2.

In the paper,the Ethernet MAC and PHY is connected by reduced media independent interface(RMII).This kind of mode requires only seven data lines,but it can support $10 \mathrm{M}$ and $100 \mathrm{M}$ interface speed as MII.Clock signals are provided by an external active crystal XOSM-57. In

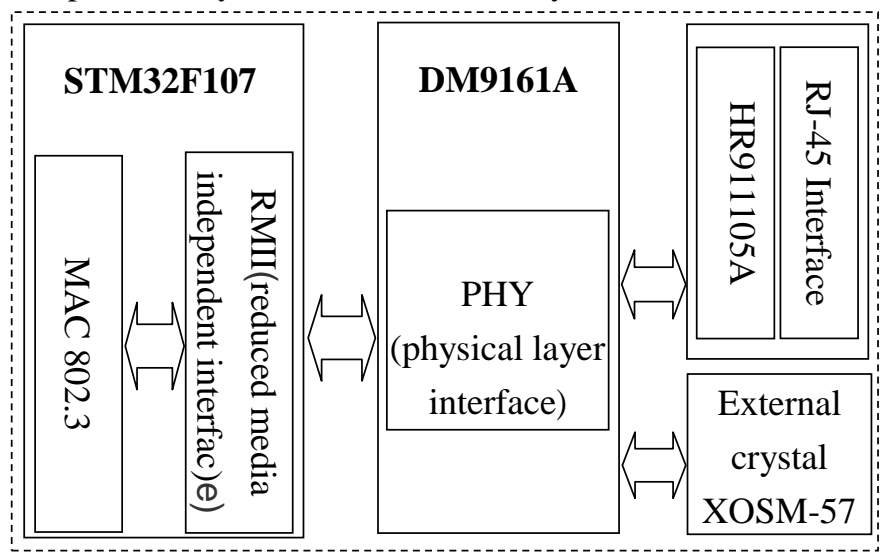

Figure 2. Block diagram of the network communication module addition,in this module,HR911105A is a 1:1 transformer chip which is used for the separation of RJ45, and it can provide electrical insulative protection for PHY,so the LAN can be in insulated environment.

\section{B. Laser and galvo scanner module hardware design}

The module is the execution part of the laser and galvo scanner control.It is mainly composed of laser generator driving circuit and laser galvo scanner driving circuit.Its hardware circuits are shown in Fig .3.

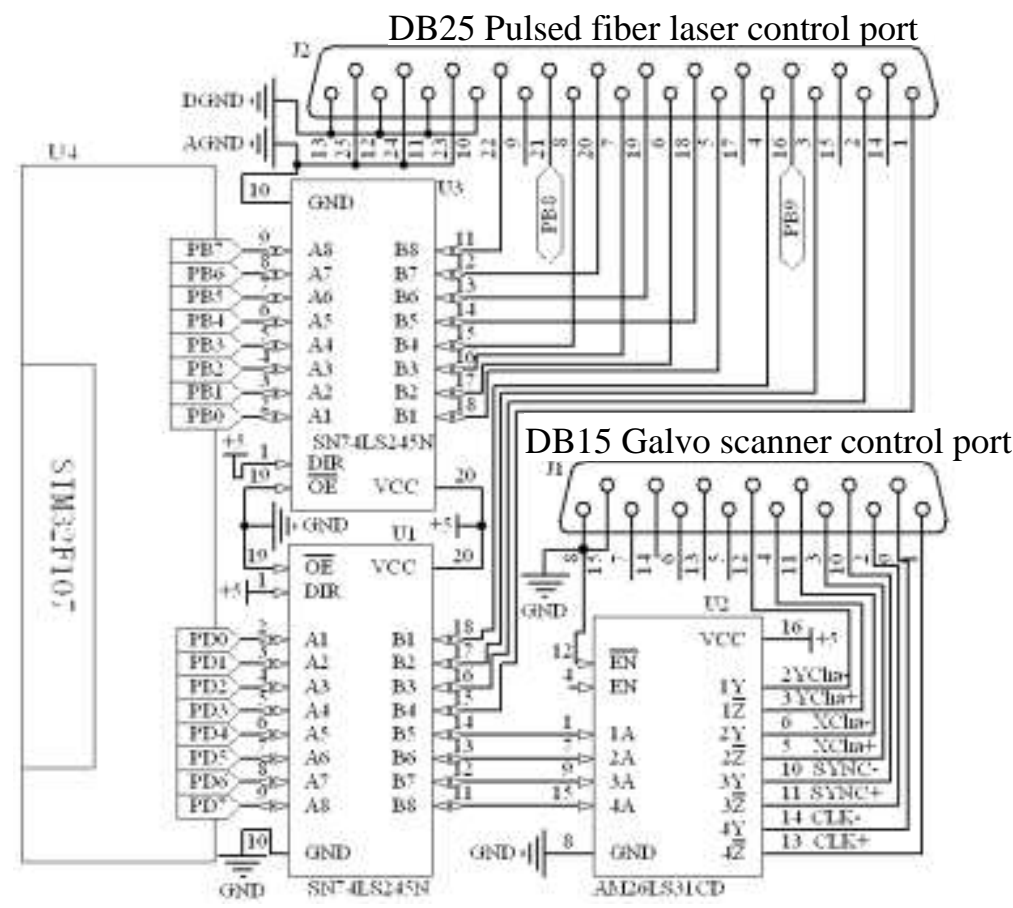

Figure 3. Laser and galvo scanner module hardware circuit diagram 
In this module,the laser generator is directly controlled by the STM32F107.It is connected by the control signals PA5-PA8.The signals that comes from 74LS245N bus driver and it then accesses to laser generator through DB25 port.The main control function and configuration analysis were as follow tables:

The control of galvo scanner part is mainly realized by the laser galvo scanner motion control module.The system produce 4 channels of 16 bit digital control

TABLE I.CONTROL FUNCTION AND CONFIGURATION ANALYSIS

\begin{tabular}{|c|c|c|c|c|}
\hline $\begin{array}{l}\text { Function } \\
\text { name }\end{array}$ & Port & \multicolumn{2}{|c|}{ State } & Analysis \\
\hline Laser power & $\mathrm{PB} 0-\mathrm{PB} 7$ & \multicolumn{2}{|c|}{$\begin{array}{l}\text { the laser power' } 256 \text { grades } \\
\text { coutrol that is from } 00 \mathrm{H}-\mathrm{FHH}\end{array}$} & Correspond to $0-100 \%$ power ontput \\
\hline $\begin{array}{c}\text { Frequeacy } \\
\text { Modulation }\end{array}$ & PD5 & \multicolumn{2}{|c|}{ On } & $\begin{array}{l}\text { Frequency modulation signal is } \\
5 \mathrm{~V} / 50-100 \mathrm{KHz} \text { pulse signals }\end{array}$ \\
\hline Laser switch & PD6 & \multicolumn{2}{|c|}{ Hidh/low leve } & Laser switch is ou off \\
\hline Mo switch & PD7 & \multicolumn{2}{|c|}{ High low leve } & MO is on off \\
\hline \multirow{5}{*}{$\begin{array}{l}\text { Alarui } \\
\text { signals }\end{array}$} & \multirow{5}{*}{$\begin{array}{l}\text { PB8, } \\
\text { PB9 }\end{array}$} & PB8 & PB9 & \\
\hline & & 0 & 0 & Laser temperiture abmormal alam \\
\hline & & 0 & 1 & Hidh reflection alam \\
\hline & & 1 & 0 & Laser waster oscillator alam \\
\hline & & 1 & 1 & Normal operation state reflection \\
\hline
\end{tabular}

signals according to the TD-XY2-100 agreement.These signals first go through a bus driver chip and it then becomes 8 channels of differential signals after going through AM26LS31CD differential drive chip.Finally, the processed signals reaches the galvo scanner control input interface(DB15).As we know,galvo scanner part integrated a galvo scanner motor driver board in its interior,so it could realize the $2^{\wedge} 16$ levels closed loop control of swing within $-10^{\circ} \sim 10^{\circ}$ according to the 8 channels of digital differential signals that we provided before.

\section{SYSTEM SOFTWARE DESIGN}

The software part mainly completes the transplantation of LwIP protocol in uC/OS-II,the initialization of the LwIP configurations, the initialization of each port in underlying devices in laser and galvo scanner module,the establishment of processing threads that is used for receiving communication data and system states and other works. The system work flow chart is shown in Fig .4.

\section{A. LwIP initialization}

The initialization of LwIP protocol stacks can be achieved by calling LwIP_Init() function.Furthermore,the data link layer and physical layer driver can be designed in accordance with the protocol stack frame.Its initialization functions are as follows.

void LwIP_Init(void)

\{

struct ip_addr ipaddr;

struct ip_addr netmask;

struct ip_addr gw;

uint8_t macaddress[6] $=\{0 \times 10 c, 0 \times b b, 0 \times a a, 0 \times 99,0 \times 88,0 \times 1$ ;

/*Initialization of dynamic memory stack*/

mem_init();

memp_init();

\# if LWIP_DHCP

ipaddr.addr $=0 ;$ netmask $\cdot$ addr $=0 ;$ gw.addr $=0$; if(!STM_EVAL_PBGetState(Button_KEY))

\{

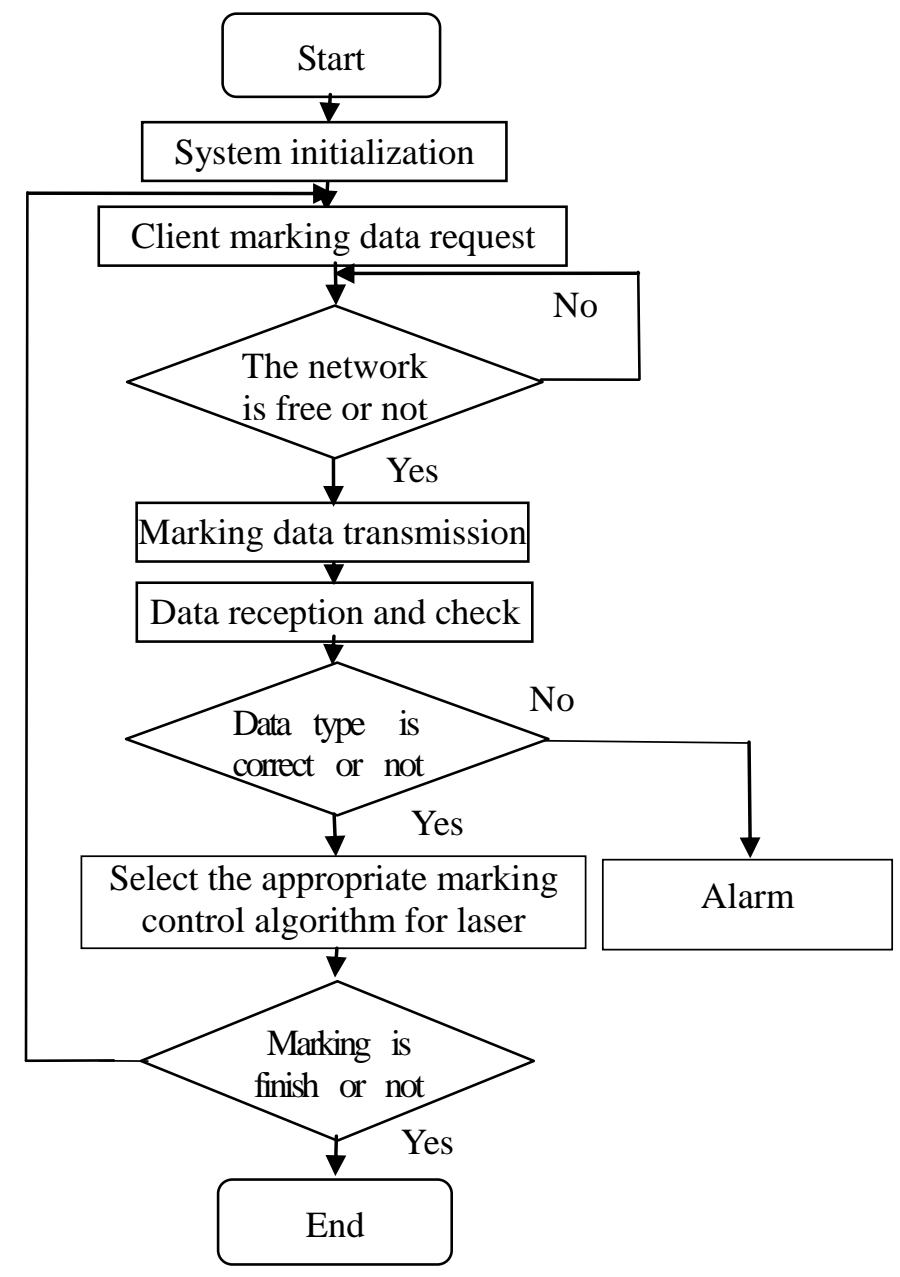

Figure 4. The system work flow diagram

macaddress[5]=CLIENTMAC6;

/*select a client*/

Server = NOT_SELECTED; $\}$

/*select a server*/

else $\{$ Server $=$ SELECTED; $\}$

\# else

IP4_ADDR(\&ipaddr, 192, 168, 1, 6);

IP4_ADDR(\&netmask, 255, 255, 255, 0);

IP4_ADDR(\&gw, 192, 168, 1, 1);

\# endif

/*Set MAC address*/

Set_MAC_Address(macaddress);

netif_add(\&netif,\&ipaddr,\&netmask,\&gw,NULL, \&ethernet /*Record the default network interface*/

if_init,\&ethernet_input);

netif_set_default(\&netif); 
//Registered network interface

$/ *$ Create a new DHCP client interface when the first call comes*/

\# if LWIP_DHCP

/*Start DHCP*/

dhcp_start(\&netif);

\# endif

/*Enable the interface of network processing*/ netif_set_up(\&netif); \}

\section{B. Underlying marking equipment port initialization}

The initialization of laser generator power control ports are mainly used for APB2 clock bus,pin memory address,port output mode,clock frequency and other parameters of the configuration.Its initialization functions are as follows, while the initialization of galvo scanner control ports are similar to it.

void LASER_Init(void)

\{

GPIO_InitTypeDef GPIO_InitStructure;

/*Enable corresponding Clock*/

RCC_APB2PeriphClockCmd(RCC_APB2Periph_GPIOB

,ENABLE);

/*Initialization GPIO_PB pinSet to a push-pull output*/ GPIO_InitStructure.GPIO_Pin=GPIO_Pin_0|GPIO_Pin1| GPIO_Pin_2|GPIO_Pin_3|GPIO_Pin_4|GPIO_Pin_5|

GPIO_Pin_6|GPIO_Pin_7;

/*Set a push-pull output*/

GPIO_InitStructure.GPIO_Mode=GPIO_Mode_Out_PP;

l*The maximum output rate is $50 \mathrm{MHz}^{*} /$

GPIO_InitStructure.GPIO_Speed

GPIO_Speed_50MHz;

/*Call the GPIO initialization function*/

GPIO_Init(GPIOD,\&GPIO_InitStructure); \}

\section{Marking data transmission}

In order to meet the need of marking technology,the establishment of data receiving threads has to complete data extraction from the frame of MAC,and then it will be processed by the LwIP data processing threads when it is transmitted to there.

In addition, we should give underlying hardware the data packets that upper layer prepares before.The underlying hardware module encapsulates data into MAC frames, and then the MAC frames are sent to data link layer and physical layer network.

void LwIP_Pkt_Handle(void) \{

$1 *$ Read a received packet from the Ethernet buffers and send it to the lwIP for handling*/ ethernetif_input(\&netif);

\}

void ethernetif_input(struct netif*netif)

\{

/*define the type of common mistakes*/

err_t
$/ *$ define the receive buffer pointer*/

struct pbuf*p;

$/ *$ move received packet into a new pbuf*/ p=low_level_input(netif);

/*no packet could be read, silently ignore this*/ if $(\mathrm{p}==\mathrm{NULL})$ return ERR_MEM; err=netif->input(p,netif); if(err!=ERR_OK)

$$
\{
$$

LWIP_DEBUGF(NETIF_DEBUG,("ethernetif_ input:IP input errorln"));

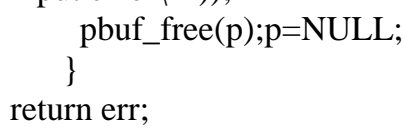

\section{CONCLUSION}

We conducted a data transmission and marking experiment according to the above analysis.Experiment results show that the system can successfully receive marking data,and it can eventually realize data elementary processing and laser engraving.Compared to the current laser marking equipment control system,the composition of scheme has obvious advantages on some aspects,such as better stability and more economical.There is important significance to the development of laser marking in the direction of integration and network.

\section{ACKNOWLEDGMENT}

All the research works are supported by the scientific research project of education department of Hubei province under granted No. D20141601, the key fund of wuhan textile university under granted No. 133037 and China industry and the Ministry of information industry under granted No. [2013]16.

\section{REFERENCES}

[1]Adam Dunkels.Design and Implementation of the LwIP TCP/IP Stack[M].Swedish:Swedish Institute of Computer Science,Inc 2001

[2]Anonymous.LASER MARKER DESIGNED FOR HARSH ENVIRONMENTS[J]. Modern Machine Shop . 2013 (9)

[3]Skeie $\mathrm{T}$, Johannessen S, Brunner C. Ethernet in substation automation[J]IEEE Control Systems Magazine,2002, 22(3): 43-51.

[4]V.F.Duma.Command functions of open loop galvanometer scanners with optimized duty cycles[J].Theoretical\&Applied Mechanics Letters, 2014(4)

[5]MING Fei Chen,Yupin Chen,Shiyuan Wu Chunwei Hu Zhipenggu.A scribing laser marking system using DSP controller[J].Optics and Lasers in Engineering . 2008 (5)

[6]CHEN Jiwei,XIANG Juwei,ZHANG Yao, Based on the STM32F107 Embedded Controller a Design of Industrial Ethernet Interface $[\mathrm{J}]$.Development \& Innovation of Machinery\&Electrical Products.2013(01):148-150

[7]ZHAO Jinrong,Wang Haifeng.Implementation of substation automation system based on the embedded Ethernet technology. Automaton of Electric Power System . 2004

[8]WANG Wenyi;LU Yong;CHEN Qingshan;KONG Fan-hui;The Design And Implementation of Galvanometer Control Board Based on

XY2-100Protocol[J].Automation\&Instrumentation,2014

(12):147-151 
[9]K.Venkatakrishnan,BTan,B.K.A Ngoi.Two-axis-scanning laser Doppler vibrometer for precision engineering[J]. Optics and Lasers in Engineering . 2002 (3)
[10]WAN Jiafu1,LI Di,ZHANG Ping,Key Technology of Embedded System Implementation for Software-based CNC System[J].Journal of Mechanical Engineering,2010(2):217-224 